\title{
National Practice Patterns for Clinical T1N0 Nasopharyngeal Cancer in the Elderly: A National Cancer Data Base Analysis
}

\author{
CARL M. POST ${ }^{1}$, CHI LIN ${ }^{1}$, SEBASTIAN ADEBERG ${ }^{2}$, \\ MRIGANK GUPTA $^{3}$, WEINING ZHEN $^{1}$ and VIVEK VERMA ${ }^{1}$ \\ ${ }^{I}$ Department of Radiation Oncology, University of Nebraska Medical Center, Omaha, NE, U.S.A.; \\ ${ }^{2}$ Department of Radiation Oncology, University Hospital Heidelberg, Heidelberg, Germany; \\ ${ }^{3}$ School of Medicine, University of Missouri Kansas City, Kansas City, MO, U.S.A.
}

\begin{abstract}
Background: The standard of care for TINO nasopharyngeal cancer $(N P C)$ is definitive radiation therapy $(R T)$. However, practice patterns in the elderly may not necessarily follow national guidelines. Herein, we investigated national practice patterns for TINO NPC. Materials and Methods: The National Cancer Data Base (NCDB) was queried for clinical T1NO primary NPC cases (2004-2013) in patients $\geq 70$ years old. Patient, tumor, and treatment parameters were extracted. Kaplan-Meier analysis was used to compare overall survival (OS) between patients receiving $R T$ versus those under observation. Logistic regression was used to examine variables associated with receipt of RT. Cox proportional hazards modeling determined variables associated with OS. Landmark analysis of patients surviving 1 year or more was performed to assess survival differences between groups. Results: In total, data of 147 patients were analyzed. $R T$ was delivered to 89 patients $(61 \%)$, whereas 58 (39\%) patients underwent observation. On multivariable analysis, older patients were less likely to receive $R T$ ( $p=0.003$ ), but there were no differences between groups in terms of Charlson-Deyo comorbidity index. Median and 5-year OS in patients receiving $R T$ versus those under observation were 71 and 33 months, and $59 \%$ and $48 \%$ $(p=0.011)$, respectively. For patients surviving 1 year or more $(n=96)$, there was a strong trend showing that receipt of $R T$ was associated with better median and 5-year OS. Conclusion: This National Data Base analysis shows that observation is relatively common for TINO NPC in the elderly, but is associated with poorer survival.
\end{abstract}

Correspondence to: Vivek Verma, MD, Department of Radiation Oncology, Fred and Pamela Buffett Cancer Center, 505 S 45th Street, Omaha, NE 68106, USA. Tel.: +1 4025523844, Fax: +1 4105523443, e-mail: vivek333@gmail.com

Key Words: Nasopharyngeal cancer, nasopharynx, radiation therapy, observation, survival.
Cancer of the nasopharynx is relatively uncommon, comprising of $0.7 \%$ of the global cancer burden, with an estimated 86,700 new diagnoses and 50,800 deaths occurring globally in 2012 (1). In the United States, nasopharyngeal carcinoma (NPC) is rare. Ninety-two percent of new cases occur in economically developing countries, with incidence rates highest in Southeastern Asia and South Africa (1). The treatment for locally advanced NPC cases is combined chemotherapy and radiation therapy (RT) (2). However, in early-stage (T1N0) NPC, RT alone is recommended (3).

The elderly pose unique challenges to the delivery of oncological care. Clinicians factor patient age and perceived ability to tolerate RT when making treatment recommendations. Not uncommonly, less aggressive approaches, such as observation alone, are utilized in the elderly to preserve quality of life (4). A 1996 study by Pignon et al. addressed this question directly by analyzing survival and toxicity for different age groups following RT for head and neck cancer, including NPC. The study showed that there was no difference in overall survival (OS) between older and younger age groups; however, older patients were more likely to suffer grade 3-4 acute functional toxicities. Although Pignon et al. studied 185 patients aged $>70$ years (12\% of the total cohort), those with NPC were clearly an under-represented minority, and the study only included one patient with T1N0 disease (5).

The aforementioned study also quantified the prevalence of RT toxicities for head and neck cancer. Older adults more commonly have impaired swallowing, weight loss, or need a feeding tube during concurrent chemoradiation (6). Despite guidelines that endorse RT in the T1N0 NPC population, it is possible that observation may be utilized, potentially related to iatrogenic side-effects affecting quality of life. The goal of this study was to use a large national data base to evaluate patterns of care in the United States for the relatively uncommon circumstance of T1N0 NPC in the elderly. 


\section{Materials and Methods}

The National Cancer Data Base (NCDB) is a joint project of the Commission on Cancer of the American College of Surgeons and the American Cancer Society. It consists of de-identified information regarding tumor characteristics, patient demographics, and patient survival for approximately $70 \%$ of the US population (7-13). The NCDB undergoes annual data integrity checks, as well as evaluations of each participating hospital's data collection processes to ensure that all data is accurate (7). The NCDB contains information not included in the Surveillance, Epidemiology, and End Results data base, including details regarding use of systemic therapy. The data used in the study were derived from a de-identified NCDB file. The American College of Surgeons and the Commission on Cancer have not verified and are neither responsible for the analytic or statistical methodology employed nor the conclusions drawn from these data by the investigators. As all patient information in the NCDB is deidentified, this study was exempt from Institutional Review Board evaluation.

Patients were drawn from the NCDB Participant User File (20042013) corresponding to newly-diagnosed NPC. The study population consisted of patients with biopsy-confirmed NPC older than 70 years of age. Patients who underwent pharyngectomy, as well as those treated with palliative intent, were excluded (Figure 1). Additionally, any patient with unknown radiation therapy or chemotherapy receipt, as well as those treated with a total radiation dose less than $60 \mathrm{~Gy}$ (thus signifying non-definitive RT), were excluded from this study (Figure 1). In accordance with the variables in the NCDB files, information collected on each patient broadly included demographic, clinical, and treatment data.

All statistical tests were performed with SAS 9.4 software (Cary, NC, USA); tests were two-sided, with a threshold of $p<0.05$ for statistical significance. Univariate and multivariate logistic regression were used to assess differences in patient, tumor, and treatment characteristics between cases who received RT versus those observed only. Upon discovery that a substantial proportion of patients were observed and underwent neither RT nor chemotherapy, univariate and multivariate logistic regression was used to determine which characteristics were associated with receipt of RT. All variables listed were considered for inclusion in models for stepwise selection. Survival analysis (performed using KaplanMeier methodology) evaluated OS, defined as the interval between the date of diagnosis and the date of death, or censoring at last contact. Endpoints such as local control and cancer-specific survival are not recorded in the NCDB. Cox proportional hazards modeling was utilized to evaluate factors predictive of OS. The proportional hazards assumption in the Cox models were met. Lastly, landmark analysis of patients surviving 1 year or more was performed to assess survival differences between both groups. This was aimed to eliminate patients with comorbidities or other factors who would not be expected to survive past a fixed amount of time. Although longer time frames could be utilized, the lower sample sizes of patients surviving up to later time periods precluded a statistically robust analysis.

\section{Results}

In total, 147 patients from the NCDB met study criteria (Figure 1). Table I displays notable clinical characteristics of the analyzed patients. A total of $89(61 \%)$ patients underwent

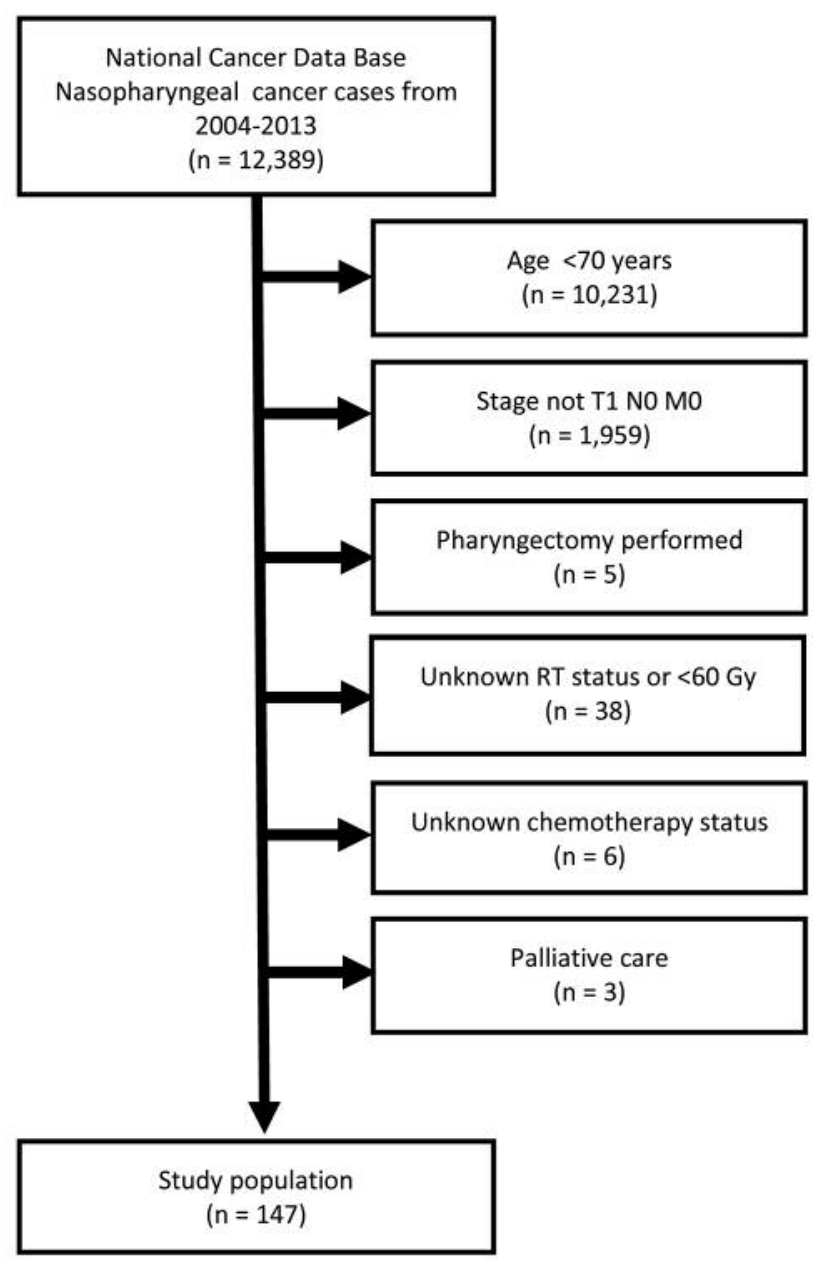

Figure 1. Patient selection diagram. RT: Radiotherapy.

RT, whereas 58 (39\%) were observed. The majority of patients were Caucasian, male, and lived in metropolitan areas. After univariate logistic regression was performed to assess for factors associated with RT receipt, multivariate logistic regression revealed that older patients were less likely to receive RT (odds ratio $(\mathrm{OR})=0.785,95 \%$ confidence interval $(\mathrm{CI})=0.659-0.905, p=0.003)$. Charlson-Deyo comorbidity index, socioeconomic factors, geographical factors, and tumor size were not significantly different between the two groups.

Kaplan-Meier estimates comparing OS in patients undergoing RT versus those undergoing observation only are illustrated in Figure 2A. In patients treated with RT, the median OS was 71 months (95\% CI=30-91 months), with 3and 5-year OS of $59 \%$ and $56 \%$, respectively. In patients who underwent observation only, median OS was 33 months (range $=17-54$ months), 3-year OS was $48 \%$, and 5-year OS was $32 \%$. There was a statistically significant improvement in OS when RT was delivered $(p=0.011)$. 

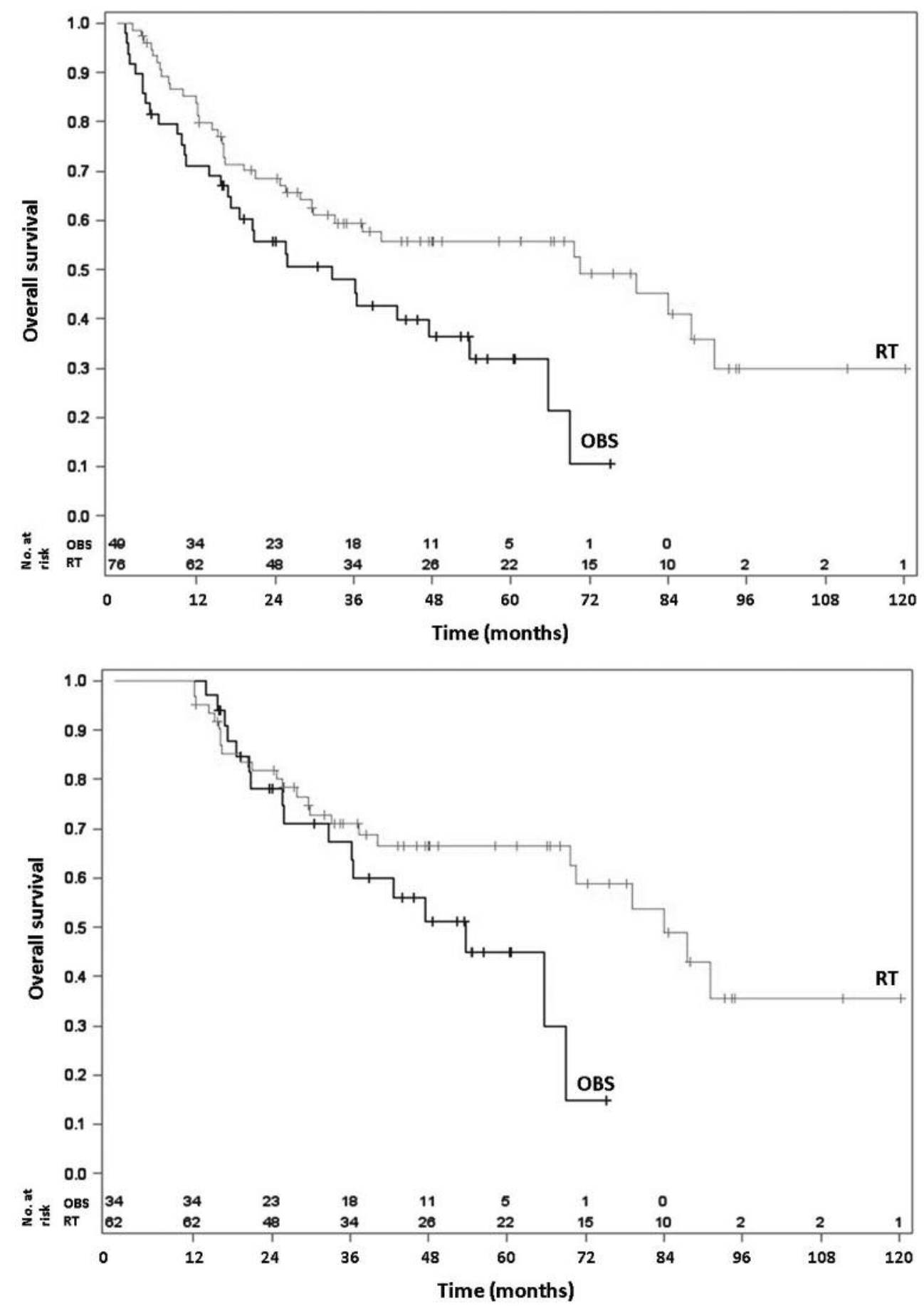

Figure 2. A: Kaplan-Meier curve comparing overall survival of patients treated with radiation therapy (RT) versus those under observation (OBS) (log-rank $p=0.011)$. B: Landmark analysis between groups that survived for at least 12 months (log-rank $p=0.072)$.

Independent predictors of OS by univariate and multivariate analysis are shown in Table II. Two prominent variables were predictive after multivariate adjustment for potential confounding factors. Increasing age (hazard ratio $=1.065,95 \% \mathrm{CI}=1.017-1.116, p=0.006$ ) predicted poorer OS. Most notably, administration of RT independently predicted improved OS (hazard $\operatorname{ratio}(\mathrm{HR})=0.583,95 \%$ $\mathrm{CI}=0.351-0.970, p=0.038$ ). 
Table I. Characteristics of the overall cohort and factors associated with receiving radiation therapy (RT).

\begin{tabular}{|c|c|c|c|c|c|c|}
\hline \multirow[t]{2}{*}{ Parameter, $\mathrm{N}(\%)$ or median (range) } & \multirow[t]{2}{*}{$\mathrm{RT}(\mathrm{N}=89)$} & \multirow[t]{2}{*}{ No RT $(\mathrm{N}=58)$} & \multicolumn{2}{|c|}{ Univariate } & \multicolumn{2}{|c|}{ Multivariate } \\
\hline & & & OR $(95 \% \mathrm{CI})$ & $p$-Value & OR $(95 \% \mathrm{CI})$ & $p$-Value \\
\hline \multicolumn{7}{|l|}{ Age (years) } \\
\hline Median (range) & $75(70-90)$ & $77(70-90)$ & & & & \\
\hline$\leq 75$ & $50(56 \%)$ & $26(45 \%)$ & 1 & REF & 1 & REF \\
\hline$>75$ & $39(44 \%)$ & $32(55 \%)$ & $0.634(0.324-1.230)$ & 0.179 & $0.785(0.659-0.905)$ & 0.003 \\
\hline \multicolumn{7}{|l|}{ Gender } \\
\hline Male & $63(71 \%)$ & $36(62 \%)$ & $1.481(0.733-2.988)$ & 0.272 & $1.152(0.280-4.552)$ & 0.840 \\
\hline Female & $26(29 \%)$ & $22(38 \%)$ & 1 & REF & 1 & REF \\
\hline \multicolumn{7}{|l|}{ Race } \\
\hline Black & $11(12 \%)$ & $7(12 \%)$ & 1 & REF & 1 & REF \\
\hline White & $62(70 \%)$ & $46(79 \%)$ & $0.858(0.296-2.349)$ & 0.768 & $0.101(0.006-0.973)$ & 0.069 \\
\hline Other & $16(18 \%)$ & $4(7 \%)$ & $2.545(0.616-11.79)$ & 0.206 & $2.867(0.104-130.5)$ & 0.545 \\
\hline Unknown & $0(0 \%)$ & $1(2 \%)$ & - & - & - & - \\
\hline \multicolumn{7}{|l|}{ Charlson-Deyo Score } \\
\hline 0 & $72(81 \%)$ & $44(76 \%)$ & 1 & REF & 1 & $\mathrm{REF}$ \\
\hline 1 & $11(12 \%)$ & $11(19 \%)$ & $0.611(0.242-1.540)$ & 0.292 & $1.001(0.152-6.830)$ & 0.999 \\
\hline 2 & $6(7 \%)$ & $3(5 \%)$ & $1.222(0.306-6.020)$ & 0.784 & $0.299(0.013-9.770)$ & 0.444 \\
\hline \multicolumn{7}{|l|}{ Insurance type } \\
\hline Private & $11(12 \%)$ & $6(10 \%)$ & 1 & REF & 1 & REF \\
\hline Medicare & $74(83 \%)$ & $50(86 \%)$ & $0.807(0.263-2.266)$ & 0.692 & $4.199(0.547-40.28)$ & 0.180 \\
\hline Medicaid/other Government & $3(3 \%)$ & $1(2 \%)$ & $1.635(0.164-37.19)$ & 0.697 & - & - \\
\hline Uninsured & $0(0 \%)$ & $0(0 \%)$ & $0.545(0.019-15.48)$ & 0.687 & - & - \\
\hline Unknown & $1(1 \%)$ & $1(2 \%)$ & & & & \\
\hline \multicolumn{7}{|l|}{ Income (US dollars/year) } \\
\hline$<\$ 30,000$ & $8(9 \%)$ & $11(19 \%)$ & 1 & REF & 1 & REF \\
\hline$\$ 30,000-\$ 34,999$ & $27(30 \%)$ & $12(21 \%)$ & $3.094(1.008-9.979)$ & 0.052 & $2.947(0.250-40.20)$ & 0.393 \\
\hline$\$ 35,000-\$ 45,999$ & $22(25 \%)$ & $15(26 \%)$ & $2.017(0.663-6.380)$ & 0.221 & $3.207(0.300-49.10)$ & 0.359 \\
\hline$\geq \$ 46,000$ & $30(34 \%)$ & $20(34 \%)$ & $2.063(0.712-6.207)$ & 0.186 & $2.237(0.153-41.26)$ & 0.566 \\
\hline Unknown & $2(2 \%)$ & $0(0 \%)$ & - & - & - & - \\
\hline \multicolumn{7}{|l|}{ Location } \\
\hline Metropolitan & $70(79 \%)$ & $49(85 \%)$ & 1 & REF & 1 & REF \\
\hline Urban & $12(13 \%)$ & $6(10 \%)$ & $1.400(0.507-4.254)$ & 0.528 & $3.085(0.287-80.81)$ & 0.400 \\
\hline Rural & $0(0 \%)$ & $1(2 \%)$ & - & - & - & - \\
\hline Unknown & $7(8 \%)$ & $2(3 \%)$ & $2.450(0.564-16.91)$ & 0.276 & - & - \\
\hline \multicolumn{7}{|l|}{ Facility type } \\
\hline Community & $56(63 \%)$ & $35(60 \%)$ & 1 & REF & 1 & REF \\
\hline Academic & $33(37 \%)$ & $23(40 \%)$ & $0.897(0.455-1.778)$ & 0.753 & $0.336(0.062-1.586)$ & 0.178 \\
\hline \multicolumn{7}{|l|}{ Facility location } \\
\hline New England & $9(10 \%)$ & $1(2 \%)$ & 1 & REF & & \\
\hline Middle Atlantic & $13(14 \%)$ & $6(10 \%)$ & $0.241(0.012-1.758)$ & 0.221 & & \\
\hline South Atlantic & $14(16 \%)$ & $17(29 \%)$ & $0.092(0.005-0.574)$ & 0.032 & & \\
\hline East North Central & $22(25 \%)$ & $13(22 \%)$ & $0.188(0.010-1.175)$ & 0.132 & & \\
\hline East South Central & $8(9 \%)$ & $6(10 \%)$ & $0.148(0.007-1.131)$ & 0.107 & & \\
\hline West North Central & $5(6 \%)$ & $2(4 \%)$ & $0.278(0.011-3.617)$ & 0.341 & & \\
\hline West South Central & $3(3 \%)$ & $3(5 \%)$ & $0.111(0.004-1.236)$ & 0.099 & & \\
\hline Mountain & $1(1 \%)$ & $2(4 \%)$ & $0.056(0.001-1.045)$ & 0.074 & & \\
\hline Pacific & $14(16 \%)$ & $8(14 \%)$ & $0.194(0.010-1.333)$ & 0.152 & & \\
\hline \multicolumn{7}{|l|}{ Distance to treating facility (miles) } \\
\hline Median (range) & $6.6(0.8-119)$ & $7.9(0.4-367)$ & $0.990(0.970-1.003)$ & 0.259 & $0.977(0.920-1.028)$ & 0.395 \\
\hline \multicolumn{7}{|l|}{ Year of diagnosis } \\
\hline $2004-2010$ & $58(65 \%)$ & $34(59 \%)$ & 1 & REF & 1 & REF \\
\hline $2011-2013$ & $31(35 \%)$ & $24(41 \%)$ & $0.757(0.383-1.500)$ & 0.423 & $0.377(0.079-1.544)$ & 0.190 \\
\hline Did not graduate from high school* & & & & & & \\
\hline$\geq 21 \%$ & $13(15 \%)$ & $11(19 \%)$ & 1 & REF & 1 & REF \\
\hline $13-20.9 \%$ & $23(26 \%)$ & $19(33 \%)$ & $1.024(0.370-2.812)$ & 0.963 & $1.113(0.085-13.31)$ & 0.932 \\
\hline $7-12.9 \%$ & $33(37 \%)$ & $12(21 \%)$ & $2.327(0.823-6.686)$ & 0.111 & $10.61(0.801-171.1)$ & 0.077 \\
\hline$<7 \%$ & $18(2 \%)$ & $16(27 \%)$ & $0.952(0.330-2.723)$ & 0.927 & $1.242(0.074-21.58)$ & 0.878 \\
\hline Unknown & $2(2 \%)$ & $0(0 \%)$ & - & - & - & - \\
\hline
\end{tabular}


Table I. Continued

\begin{tabular}{|c|c|c|c|c|c|c|}
\hline \multirow[t]{2}{*}{ Parameter, N (\%) or median (range) } & \multirow[t]{2}{*}{$\mathrm{RT}(\mathrm{N}=89)$} & \multirow[t]{2}{*}{ No RT (N=58) } & \multicolumn{2}{|c|}{ Univariate } & \multicolumn{2}{|c|}{ Multivariate } \\
\hline & & & OR $(95 \% \mathrm{CI})$ & $p$-Value & OR $(95 \% \mathrm{CI})$ & $p$-Value \\
\hline \multicolumn{7}{|l|}{ Tumor size $(\mathrm{cm})$} \\
\hline Median (range) & $2.7(0.5-5)$ & $2(0.7-5)$ & $1.020(0.980-1.064)$ & 0.339 & $1.026(0.960-1.098)$ & 0.452 \\
\hline$\leq 2 \mathrm{~cm}$ & $21(24 \%)$ & $16(28 \%)$ & 1 & REF & & \\
\hline$>2-<3 \mathrm{~cm}$ & $21(24 \%)$ & $8(14 \%)$ & $1.999(0.718-5.879)$ & 0.193 & & \\
\hline$\geq 3-\leq 5 \mathrm{~cm}$ & $17(19 \%)$ & $7(12 \%)$ & $1.850(0.633-5.773)$ & 0.271 & & \\
\hline Unknown & $30(34 \%)$ & $27(46 \%)$ & $0.847(0.365-1.944)$ & 0.695 & & \\
\hline \multicolumn{7}{|l|}{ Chemotherapy } \\
\hline No & $58(65 \%)$ & $58(100 \%)$ & - & - & & \\
\hline Yes & $31(35 \%)$ & $0(0 \%)$ & - & - & & \\
\hline \multicolumn{7}{|l|}{ Radiation dose (Gy) } \\
\hline Median (range) & $70(60-90)$ & $0(0-0)$ & - & - & & \\
\hline
\end{tabular}

OR, Odds ratio; CI, confidence interval. *Of adults in the patient's zip code. Statistically significant $p$-values are in bold. Values may not add up to $100 \%$ because of rounding. Only variables in the final multivariate model are included.

Landmark analysis of patients surviving at least 1 year can be seen in Figure 2B ( $\mathrm{n}=96)$, which showed a trend towards improved median, 3-year and 5-year OS in the group receiving RT $(p=0.072)$, with values for the RT and observation groups of 84 and 43 months, 71 and 59\%, and 67 and $39 \%$, respectively.

\section{Discussion}

To our knowledge, this is the first study to define national practice patterns and survival outcomes for T1N0 NPC in the elderly population. Our study of a large national data base of this rare condition in a relatively vulnerable patient population most notably demonstrates that observation continues to be a common practice. However, this is independently associated with poorer survival. The results of this study parallel other observational studies of other earlystage neoplasms in the elderly, demonstrating the detriment of observation alone $(11,14)$.

The NCDB contains information on approximately $70 \%$ of the United States cancer population, so national practice patterns can be easily queried (7-13). Despite existing recommendations of RT for this patient population, this study shows that a strikingly high number of patients (38\%) were observed only. Age and associated comorbidity are possibly some of the reasons why observation continues to be practiced, as clinicians may believe that life expectancy is too short to warrant more aggressive care. This notion has been posited before, as a meta-analysis by Metges et al. concluded that comorbid conditions were more detrimental than head and neck cancer itself, and recommended that those in good health should receive RT (15). Our results counter those data, as receipt of RT was independently predictive of improved outcomes, recognizing that baseline comorbidity scores were equivalent between the RT and observation groups.

Furthermore, landmark analysis of patients surviving 1 year or more was also carried out to safeguard against any potential confounding and unforeseen factors that would lead to mortality before any treatment benefit would be seen. Despite removal of additional confounding factors, a trend towards superior OS with RT was still observed $(p=0.072)$. This likely was not statistically significant because over onethird of the sample size was removed with this analysis.

Interestingly, a minority of the patients (35\%) who received RT also received concurrent or adjuvant chemotherapy, despite no National Comprehensive Cancer Network recommendations for chemotherapy use in these cases. The lack of this recommendation by the National Comprehensive Cancer Network was recently confirmed by a 2017 study that observed no differences in survival between patients with T1N0 NPC treated with or without chemotherapy (16).

Although utilizing the NCDB is an effective way to study this relatively rare clinical circumstance, this investigation is not without limitations. First and foremost, it is impossible to determine the precise reasons and rationale for observation in certain patients. Thus, although there were no differences in comorbidity scores between groups, the observation cohort may have been predisposed to having poorer outcomes. Secondly, the NCDB does not collect treatment toxicity data, so our analyzed cohort may comprise a better prognostic group, while those unable to tolerate RT ( $\geq 60 \mathrm{~Gy}$ ) due to toxicity would be excluded. Thirdly, the inherent retrospective nature of NCDB studies, as well as the limited sample sizes of T1N0 cases in the elderly, make conclusions 
Table II. Univariate and multivariate Cox proportional hazards models for overall survival.

\begin{tabular}{|c|c|c|c|c|c|c|c|}
\hline \multirow[b]{2}{*}{ Parameter } & & \multicolumn{3}{|c|}{ Univariate } & \multicolumn{3}{|c|}{ Multivariate } \\
\hline & & HR & $95 \% \mathrm{CI}$ & $p$-Value & HR & $95 \% \mathrm{CI}$ & $p$-Value \\
\hline RT & Yes vs. no & 0.840 & $0.398-1.773$ & 0.648 & 0.583 & $0.351-0.970$ & 0.038 \\
\hline Age & Continuous & 1.055 & $0.991-1.123$ & 0.094 & 1.065 & $1.017-1.116$ & 0.006 \\
\hline Gender & Male $v s$. female & 0.578 & $0.272-1.227$ & 0.153 & & & \\
\hline \multirow[t]{2}{*}{ Race } & White vs. Black & 1.732 & $0.603-4.977$ & 0.308 & & & \\
\hline & Non-White non-Black $v s$. Black & 1.227 & $0.254-5.922$ & 0.799 & & & \\
\hline \multirow[t]{2}{*}{ Charlson-Deyo score } & 1 vs. 0 & 1.526 & $0.626-3.723$ & 0.353 & & & \\
\hline & 2 vs. 0 & 2.623 & $0.775-8.876$ & 0.121 & & & \\
\hline \multirow[t]{2}{*}{ Insurance } & Medicare $v s$. private & 2.480 & $0.755-8.147$ & 0.134 & & & \\
\hline & Medicaid vs. other Government vs. private & 4.409 & $0.654-29.73$ & 0.128 & & & \\
\hline \multirow[t]{3}{*}{ Income } & $\$ 30,000-34,999$ vs. $<\$ 30,000$ & 0.452 & $0.210-0.975$ & 0.043 & & & \\
\hline & $\$ 35,000-\$ 45,999$ vs. $<\$ 30,000$ & 0.449 & $0.185-1.093$ & 0.078 & & & \\
\hline & $\geq \$ 46,000$ vs. $<\$ 30,000$ & 0.526 & $0.204-1.353$ & 0.183 & & & \\
\hline Geographic location & Urban $v s$. metropolitan & 1.808 & $0.876-3.731$ & 0.109 & 1.782 & $0.937-3.389$ & 0.066 \\
\hline Facility & Academic $v s$. community & 0.606 & $0.292-1.257$ & 0.178 & & & \\
\hline \multirow[t]{7}{*}{ Facility location } & Middle Atlantic $v s$. New England & 1.498 & $0.379-5.917$ & 0.564 & & & \\
\hline & South Atlantic vs. New England & 2.463 & $0.690-8.795$ & 0.165 & & & \\
\hline & East North Central vs. New England & 2.898 & $0.791-10.62$ & 0.108 & & & \\
\hline & East South Central vs. New England & 2.259 & $0.521-9.796$ & 0.276 & & & \\
\hline & West North Central vs. New England & 2.037 & $0.359-11.56$ & 0.422 & & & \\
\hline & West South Central vs. New England & 3.396 & $0.661-17.45$ & 0.143 & & & \\
\hline & Pacific $v s$. New England & 1.741 & $0.332-9.134$ & 0.512 & & & \\
\hline Distance to treatment facility & Continuous & 0.996 & $0.979-1.013$ & 0.645 & & & \\
\hline \multirow[t]{3}{*}{ Did not graduate from high school* } & $13-20.9 \%$ vs. $\geq 21 \%$ & 0.770 & $0.359-1.648$ & 0.500 & & & \\
\hline & $7-12.9 \%$ vs. $\geq 21 \%$ & 0.612 & $0.262-1.428$ & 0.256 & & & \\
\hline & $<7 \%$ vs. $\geq 21 \%$ & 0.957 & $0.357-2.566$ & 0.930 & & & \\
\hline Chemotherapy & Yes vs. no & 0.937 & $0.406-2.161$ & 0.878 & & & \\
\hline Tumor size & Continuous & 0.983 & $0.939-1.030$ & 0.472 & & & \\
\hline
\end{tabular}

HR, Hazard ratio; CI, confidence interval. *Of adults in the patient's zip code. Statistically significant $p$-values are in bold. Only statistically significant or near-statistically significant values are shown on multivariate analysis.

less statistically sound. Next, the NCDB does not allow for an assessment of subsequent lines of treatment (e.g. reirradiation, further systemic or targeted therapy), which could influence patient outcomes. In addition, the simplified nature of 'overall survival', which does not factor in local or locoregional control, or even cancer-specific survival, may minimize the conclusions that can be drawn from this national data base. Lastly, the NCDB also does not provide details such as performance status, or RT field design/ volumes/techniques, that may have been significant factors that swayed clinicians to favor a certain treatment option over another.

\section{Conclusion}

Our analysis of national practice patterns and survival outcomes of elderly patients with T1N0 NPC demonstrates that rather than observation, treatment with radiation therapy is associated with higher survival and is, thus, recommended.

\section{References}

1 Torre LA, Bray F, Siegel RL, Ferlay J, Lortet-Tieulent J and Jemal A: Global cancer statistics, 2012. CA Cancer J Clin 65: 87-108, 2015.

2 Blanchard P, Lee A, Marguet S, Leclercq J, Ng WT, Ma J, Chan AT, Huang PY, Benhamou E, Zhu G, Chua DT, Chen Y, Mai HQ, Kwong DL, Cheah SL, Moon J, Tung Y, Chi KH, Fountzilas G, Zhang L, Hui EP, Lu TX, Bourhis J and Pignon JP: Chemotherapy and radiotherapy in nasopharyngeal carcinoma: an update of the MAC-NPC meta-analysis. Lancet Oncol 16: 645-655, 2015.

3 Head and Neck Cancers. NCCN Clinical Practice Guidelines in Oncology. Version 2.2017. https://www.nccn.org/professionals/ physician_gls/pdf/head-and-neck.pdf, accessed May 2017.

4 Older Adult Oncology. NCCN Clinical Practice Guidelines in Oncology. Version 2.2017. https://www.ncen.org/professionals/ physician_gls/pdf/senior.pdf, accessed May 2017.

5 Pignon T, Horiot JC, Van den Bogaert W, Van Glabbeke $M$ and Scalliet P: No age limit for radical radiotherapy in head and neck tumours. Eur J Cancer 32A: 2075-2081, 1996. 
6 Verma V and Ganti AK: Concurrent chemoradiotherapy in older adults with squamous cell head and neck cancer: Evidence and management. J Geriatr Oncol 7: 145-53, 2016.

7 Bilimoria KY, Stewart AK, Winchester DP and Ko CY: The National Cancer Data Base: A powerful initiative to improve cancer care in the United States. Ann Surg Oncol 15: 683-690, 2008.

8 Stahl JM, Corso CD, Verma V, Park HS, Nath SK, Husain ZA, Simone CB 2nd, Kim AW and Decker RH: Trends in stereotactic body radiation therapy for stage I small cell lung cancer. Lung Cancer 103: 11-16, 2017.

9 Verma V, McMillan MT, Grover S and Simone CB 2nd: Stereotactic body radiation therapy and the influence of chemotherapy on overall survival for large ( $\geq 5$ centimeter) nonsmall cell lung cancer. Int J Radiat Oncol Biol Phys 97: 146154,2017

10 Haque W, Verma V, Butler EB and Teh BS: Patterns of care and outcomes of multi-agent versus single-agent chemotherapy as part of multimodal management of low-grade glioma. J Neurooncol 133: 369-375, 2017.

11 Moreno AC, Verma V, Hofstetter WL and Lin SH: Patterns of care and treatment outcomes of elderly patients with stage I esophageal cancer: Analysis of the National Cancer Data Base. J Thorac Oncol 12: 1152-1160, 2017.

12 Haque W, Verma V, Butler EB and Teh BS: Definitive chemoradiation at high-volume facilities is associated with improved survival in glioblastoma. J Neurooncol 135: 173-181, 2017
13 McMillan MT, Ojerholm E, Verma V, Higgins KA, Singhal S, Predina JD, Berman AT, Grover S, Robinson CG and Simone CB 2nd: Radiation treatment time and overall survival in locally advanced non-small cell lung cancer. Int J Radiat Oncol Biol Phys 98: 1142-1152, 2017.

14 Nanda RH, Liu Y, Gillespie TW, Mikell JL, Ramalingam SS, Fernandez FG, Curran WJ, Lipscomb J and Higgins KA: Stereotactic body radiation therapy versus no treatment for early stage non-small cell lung cancer in medically inoperable elderly patients: A National Cancer Data Base analysis. Cancer 121: 4222-4230, 2015.

15 Metges JP, Eschwege F, de Crevoisier R, Lusinchi A, Bourhis J and Wibault P: Radiotherapy in head and neck cancer in the elderly: a challenge. Crit Rev Oncol Hematol 34: 195-203, 2000.

16 Verma V, Ryckman JM, Simone CB 2nd and Lin C: Patterns of care and outcomes with the addition of chemotherapy to radiation therapy for stage I nasopharyngeal cancer. Acta Oncol 57: $257-261,2018$

Received December 21, 2017

Revised January 28, 2018

Accepted January 30, 2018 